\title{
Effectiveness of Interventions to Control Transmission of Reemergent Cases of COVID-19 — Jilin Province, China, 2020
}

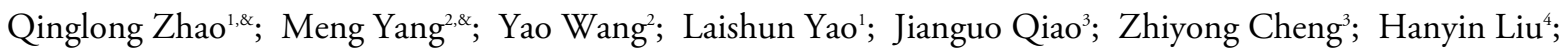 \\ Xingchun $\mathrm{Liu}^{2}$; Yuanzhao Zhu'² Zeyu Zhao'; Jia Rui²; Tianmu Chen ${ }^{2, *}$
}

\section{Summary}

What is already known about this topic?

COVID-19 has a high transmissibility calculated by mathematical model. The dynamics of the disease and the effectiveness of intervention to control the transmission remain unclear in Jilin Province, China. What is added by this report?

This is the first study to report the dynamic characteristics and to quantify the effectiveness of interventions implemented in the second outbreak of COVID-19 in Jilin Province, China. The effective reproduction number of the disease before and after May 10 was 4.00 and $p<0.01$, respectively. The combined interventions reduced the transmissibility of COVID-19 by $99 \%$ and the number of cases by $98.36 \%$.

What are the implications for public health practice?

The findings of this study would add data on the transmission of COVID-19 and provide evidence to prepare the second outbreak transmission of the disease in other areas of China even in many other countries.

China has successfully controlled the first outbreak of the coronavirus disease 2019 (COVID-19) due to the strictly implemented public health policy including active case finding with case management (1). However, it has become an essential public health concern that whether there would be a second outbreak of COVID-19 in China, and how to control the second outbreak? Jilin Province, locating in the north east of China, has also controlled its first outbreak of COVID-19 successfully (2). On May 7, 2020, an outbreak of COVID-19 was reported in Shulan City, Jilin Province, China. The outbreak is the second outbreak in the province. Therefore, it has public health significance to quantify the transmissibility, to assess the effectiveness of interventions, and to provide experience for other provinces or cities in China, or even for other countries to deal with the second outbreak of COVID-19 outbreaks.

Based on our previous study (2-5), we developed a Susceptible-Exposed-Infectious-AsymptomaticRemoved (SEIAR) model to fit the data in Jilin Province and to perform the assessment. In the SEIAR model, individuals were divided into five compartments: Susceptible (S), Exposed (E), Infectious $(I)$, Asymptomatic $(A)$, and Removed $(R)$, and the equations of the model were shown as follows:

$$
\begin{gathered}
\frac{d S}{d t}=-\beta S(I+\kappa A) \\
\frac{d E}{d t}=\beta S(I+\kappa A)-p \omega^{\prime} E-(1-p) \omega E \\
\frac{d I}{d t}=(1-p) \omega E-\gamma I-f I \\
\frac{d A}{d t}=p \omega^{\prime} E-\gamma^{\prime} A \\
\frac{d R}{d t}=\gamma I+\gamma^{\prime} A
\end{gathered}
$$

There are eight parameters $\left(\beta, \kappa, \omega, \omega^{\prime}, p, \gamma\right.$, $\gamma^{\prime}$, and $f$ ) in the model. The transmission rate, $\beta$, was estimated by fitting the reported data. Since only limited secondary transmission was observed due to $A$, in this study, we assumed that the transmissibility of $A$ was $5 \%$ of that of $I$. Therefore, the parameter $\kappa$, the relative transmissibility coefficient of $A$ compared with $I$, was set as 0.05 in this study. According to the reported data in the outbreak, we investigated the following parameters: A) the incubation period $(1 / \omega)$ and the latent period $\left(1 / \omega^{\prime}\right)$ was 8 days and 6 days, respectively; B) the infectious periods of $A$ and $I$ were both set as 3 days; C) the parameter $p$, the proportion of $A$, was $6.52 \%$; D) and the parameter $f$, the case fatality rate, was $2.17 \%$.

Commonly, we used the basic reproduction number $\left(R_{0}\right)$ to assess the transmissibility of COVID-19. $R_{0}$ 
was defined as the expected number of secondary infections that result from introducing a single infected individual into an otherwise susceptible population(4). However, if intervention was implemented, $R_{0}$ should be replaced as effective reproduction number $\left(R_{\text {eff }}\right)$ which could be calculated by the following equation:

$$
R_{e f f}=\beta S\left(\frac{1-p}{\gamma+f}+\frac{\kappa p}{\gamma^{\prime}}\right)
$$

Berkeley Madonna 8.3.18 (developed by Robert Macey and George Oster of the University of California at Berkeley; Copyright (C) 1993-2001 Robert I. Macey \& George F. Oster, University of California, Berkeley, CA) was employed to perform the curve fitting and simulation.

The data were collected including all reported cases in Jilin Province from April 25, 2020 to June 4, 2020. The data included the basic information (sex, age, occupation, address), the classification (asymptomatic infection and confirmed cases), key date point (contact date, symptom onset date, hospitalization date, and diagnosed date), and the number of close contacts of each case.

From April 25, 2020 to June 4, 2020, a total of 43 confirmed cases and 3 asymptomatic infections were reported in the province. The epidemic peak of outbreak was during May 8 to May 10 (Figure 1). The outbreak lasted 7 generations. The secondary attack rate (TAR) of the index case and its following generations was $40.00 \%, 2.59 \%, 4.55 \%, 5.09 \%$, $1.19 \%$, and $0.55 \%$, respectively. Based on the information of some cases which had the exact exposure date and symptoms onset date, the median incubation period of the cases was calculated as 6 days (range: 2-11 days). The epidemic spread to five districts and cities in Jilin Province (Shulan, Fengman, Chuanying, Changyi, and Gaoxin). About $48.84 \%$ confirmed cases had an age of 25-46 years. The main occupation of patients was housework and unemployment, cadres and staff, and business services (Table 1).

The SEIAR model fitted the data well $\left(R^{2}=0.29\right.$, $p<0.01)$. The value of $R_{\text {eff }}$ before and after May 10 was 4.00 and $p<0.01$, respectively. Therefore, the combined interventions reduced the transmissibility of COVID-19 by $99 \%$ in the area (Figure 1). According to the simulation results, if the comprehensive intervention measures were not taken on May 10, as of June 4 , the predicted cumulative number of cases would be 2,833 .

Three further scenarios were simulated as follows: Scenario A: the duration from onset to diagnosed date was shortened by $50 \%$ after May 10; Scenario B: the value of $R_{\text {eff }}$ was shortened by $50 \%$ after May 10; Scenario C: all the cases (exception asymptomatic infections) were isolated after May 10. The results showed that: under the circumstance of Scenario A, the number of cumulative cases would be 503 with a reduction of $82.24 \%$; under the circumstance of Scenario B, the number of cumulative cases would be 309 with a reduction of $89.09 \%$; under the circumstance of Scenario C, the number of cumulative cases would be 211 with a reduction of $92.55 \%$. The reported cumulative number of cases was 46 (43

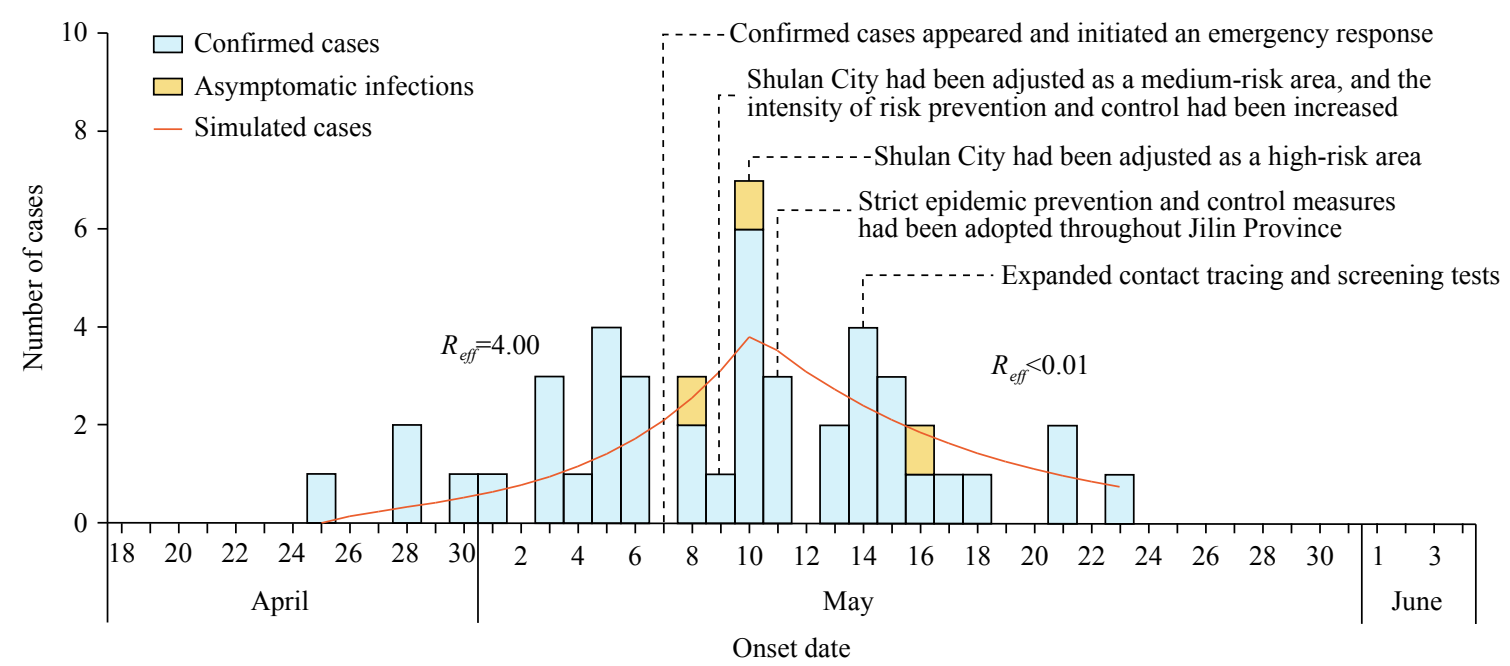

FIGURE 1. Curve fitting results of Susceptible-Exposed-Infectious-Asymptomatic-Removed (SEIAR) model to fit the data of COVID-19 cases in Jilin Province, China. 
TABLE 1. The epidemiological characteristics of 46 COVID-19 cases or infections in Jilin Province, China.

\begin{tabular}{|c|c|c|c|c|}
\hline \multirow{2}{*}{ Variables } & \multicolumn{2}{|c|}{ Confirmed cases } & \multicolumn{2}{|c|}{ Asymptomatic infections } \\
\hline & $\mathbf{n}$ & $\%$ & $\mathbf{n}$ & $\%$ \\
\hline \multicolumn{5}{|l|}{ Areas } \\
\hline Shulan City in Jilin City & 20 & 46.51 & 0 & 0.00 \\
\hline Fengman District in Jilin City & 16 & 37.21 & 2 & 66.67 \\
\hline Chuanying District in Jilin City & 3 & 6.98 & 0 & 0.00 \\
\hline Changyi District in Jilin City & 1 & 2.32 & 0 & 0.00 \\
\hline Gaoxin District in Jilin City & 3 & 6.98 & 0 & 0.00 \\
\hline Kuancheng District in Changchun City & 0 & 0.00 & 1 & 33.33 \\
\hline \multicolumn{5}{|l|}{ Sex } \\
\hline Male & 19 & 44.19 & 1 & 33.33 \\
\hline Female & 24 & 55.81 & 2 & 66.67 \\
\hline \multicolumn{5}{|l|}{ Age (years) } \\
\hline$\leq 24$ & 3 & 6.98 & 2 & 66.67 \\
\hline $25-46$ & 21 & 48.84 & 1 & 33.33 \\
\hline $47-68$ & 12 & 27.90 & 0 & 0.00 \\
\hline$\geq 69$ & 7 & 16.28 & 0 & 0.00 \\
\hline \multicolumn{5}{|l|}{ Occupation } \\
\hline Housework and unemployment & 14 & 32.56 & 0 & 0.00 \\
\hline Cadres and staff & 8 & 18.60 & 1 & 33.33 \\
\hline Business services & 6 & 13.95 & 0 & 0.00 \\
\hline Retired personnel & 5 & 11.63 & 0 & 0.00 \\
\hline Farmer & 5 & 11.63 & 0 & 0.00 \\
\hline Teacher & 2 & 4.64 & 0 & 0.00 \\
\hline Scattered children & 1 & 2.33 & 2 & 66.67 \\
\hline Student & 1 & 2.33 & 0 & 0.00 \\
\hline Medical staff & 1 & 2.33 & 0 & 0.00 \\
\hline
\end{tabular}

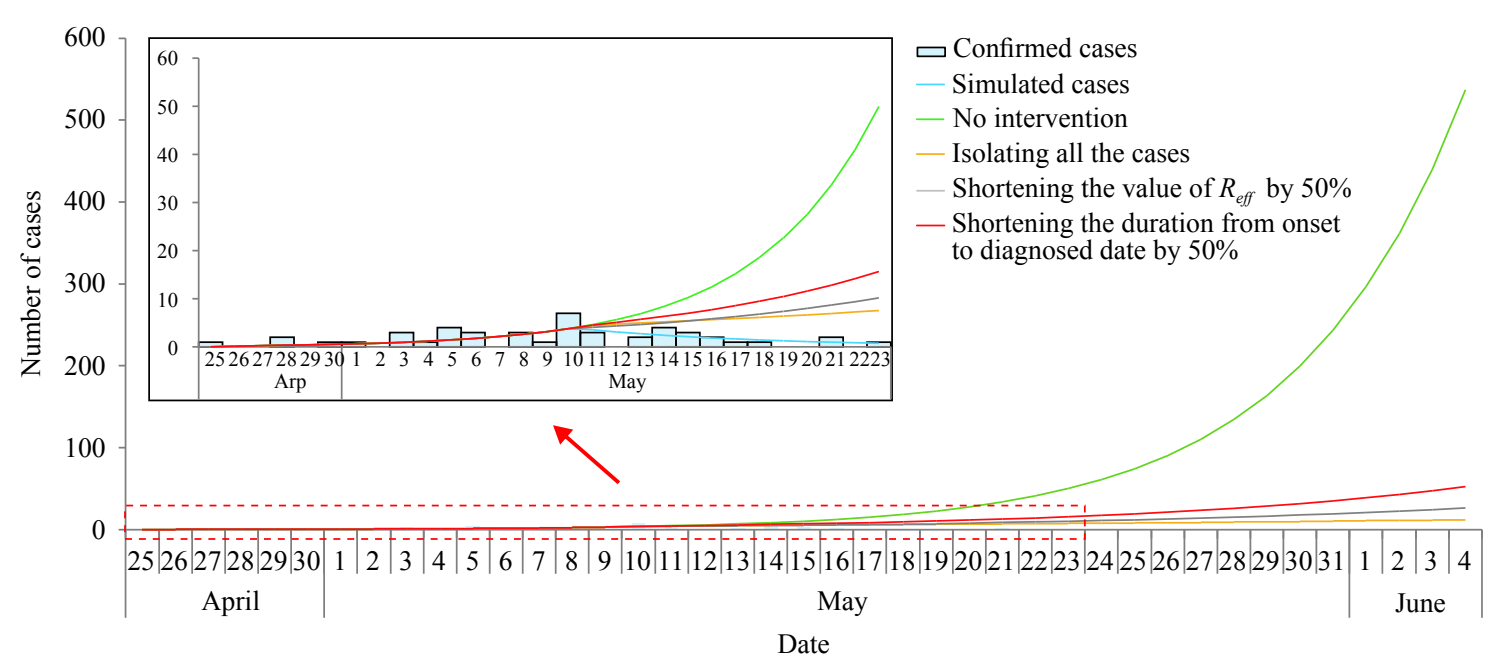

FIGURE 2. Simulating the effectiveness of interventions to control the transmission of COVID-19 in Jilin Province, China.

confirmed cases and 3 asymptomatic infection). The number of cases was reduced by $98.38 \%$ by comprehensive prevention and control measures (Figure 2). 


\section{DISCUSSION}

Most of the cases in this outbreak were clustered in Shulan City and Fengman District and transmitted through family and work contact, which shows that close contacts were the main transmission route in the spread of the outbreak. The distribution characteristics of cases in region, age and occupation further confirmed the transmission route.

The value of $R_{\text {eff }}$ was 4.00 and $p$-value was below 0.01 before and after May 10, respectively, which indicates that one case can transmit more than four new cases before May 10 and the transmissibility of COVID-19 was decreased to a low level due to the interventions implemented by the authorities. Most of the cases, which got symptoms after May 10, were probably infected before the date and were in their incubation periods.

Our three simulation scenarios showed that case finding and case isolation has the highest effectiveness followed by shortening $R_{\text {eff }}$ and the duration from onset to diagnosed date. The simulation results also showed that the comprehensive countermeasures in Jilin Province including emergency response in time, enhancing the risk level of Shulan City, and improving the ability of case finding, had reduced the number of infected people by $98.38 \%$ (Figure 2).

The successful control of this outbreak has provided a good experience to control the COVID-19 transmission in future. High transmissibility of the disease calls for a sensitive surveillance system to find out the infected people at the early stage of the transmission. It is also essential to improve the ability of local public health departments on epidemiologic field investigation, laboratory test to screen the infection in a large area, and implementing the interventions such as case isolation, wearing face mask, and keeping social distance. However, the transmission route of this outbreak was person-toperson. More researches are needed to explore the control of the other routes such as environment-toperson and food-to-person.

Acknowledgements: We thank the staffs of Centers for Disease Control and Prevention and clinics at different levels in China for assistance. We also thank the support from Undergraduate Innovation Practice Platform of School of Public Health, Xiamen University.

Conflicts of interest: No conflicts of interest were reported.

Fundings: This work was partly supported by the Bill \& Melinda Gates Foundation (INV-005834), the Science and Technology Program of Fujian Province (No: 2020Y0002), the Xiamen New Coronavirus Prevention and Control Emergency Tackling Special Topic Program (No: 3502Z2020YJ03), and the Open Research Fund of State Key Laboratory of Molecular Vaccinology and Molecular Diagnostics (SKLVD2019KF005).

doi: $10.46234 / \mathrm{ccdcw} 2020.181$

\# Corresponding author: Tianmu Chen, chentianmu@xmu.edu.cn, 13698665@qq.com.

\footnotetext{
1 Jilin Provincial Center for Disease Control and Prevention, Changchun, Jilin, China; ${ }^{2}$ State Key Laboratory of Molecular Vaccinology and Molecular Diagnostics, School of Public Health, Xiamen University, Xiamen, Fujian, China; ${ }^{3}$ Jilin Municipal Center for Disease Control and Prevention, Jilin City, Jilin Province, China; ${ }^{4}$ Shulan Center for Disease Control and Prevention, Shulan, Jilin City, Jilin Province, China.

\& Joint first authors.
}

Submitted: June 20, 2020; Accepted: August 10, 2020

\section{REFERENCES}

1. Li ZJ, Chen QL, Feng LZ, Rodewald L, Xia YY, Yu HL, et al. Active case finding with case management: the key to tackling the COVID-19 pandemic. Lancet 2020;396(10243):63 - 70. http://dx.doi.org/10.1016/ S0140-6736(20)31278-2.

2. Zhao QL, Wang Y, Yang M, Li MN, Zhao ZY, Lu XR, et al. Evaluating the effectiveness of countermeasures to control the novel coronavirus disease 2019 in Jilin Province, China. Preprint 2020 http://dx.doi.org/10.21203/rs.3.rs-26184/v1.

3. Chen TM, Rui J, Wang QP, Zhao ZY, Cui JA, Yin L. A mathematical model for simulating the phase-based transmissibility of a novel coronavirus. Infect Dis Poverty 2020;9(1):18 - 25. http://dx.doi.org/ 10.1186/s40249-020-00640-3.

4. Lao XY, Luo L, Lei Z, Fang T, Chen Y, Liu YH, et al. Epidemiological characteristics and the effectiveness of countermeasures to control coronavirus disease 2019 in Ningbo City, China. Preprint 2020. http://dx.doi.org/10.21203/rs.3.rs-26311/v1.

5. Zhao ZY, Zhu YZ, Xu JW, Hu QQ, Lei Z, Rui J, et al. A mathematical model for estimating the age-specific transmissibility of a novel coronavirus. medRxiv 2020. http://dx.doi.org/10.1101/2020.03.05. 20031849. 